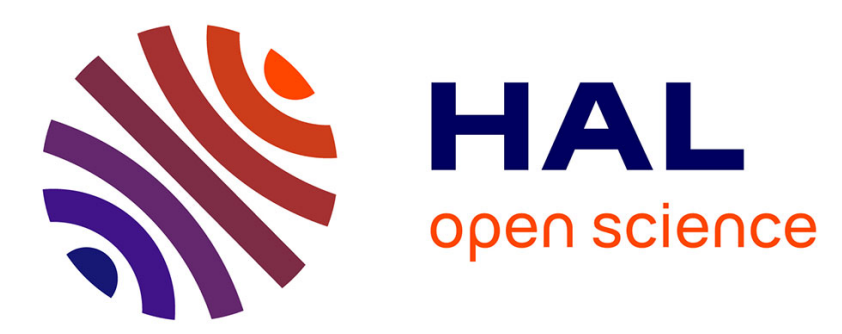

\title{
Moving droplets on asymmetrically structured surfaces
} Olivier Sandre, L. Gorre-Talini, Armand Ajdari, Jacques Prost, Pascal Silberzan

\section{To cite this version:}

Olivier Sandre, L. Gorre-Talini, Armand Ajdari, Jacques Prost, Pascal Silberzan. Moving droplets on asymmetrically structured surfaces. Physical Review E , 1999, 60 (3), pp.2964-2972. 10.1103/PhysRevE.60.2964 . hal-02168818

\section{HAL Id: hal-02168818 https://hal.science/hal-02168818}

Submitted on 2 Nov 2019

HAL is a multi-disciplinary open access archive for the deposit and dissemination of scientific research documents, whether they are published or not. The documents may come from teaching and research institutions in France or abroad, or from public or private research centers.
L'archive ouverte pluridisciplinaire HAL, est destinée au dépôt et à la diffusion de documents scientifiques de niveau recherche, publiés ou non, émanant des établissements d'enseignement et de recherche français ou étrangers, des laboratoires publics ou privés. 


\title{
Moving droplets on asymmetrically structured surfaces
}

\author{
O. Sandre, ${ }^{1}$ L. Gorre-Talini, ${ }^{1, *}$ A. Ajdari, ${ }^{2}$ J. Prost,${ }^{1}$ and P. Silberzan ${ }^{1}$ \\ ${ }^{1}$ Institut Curie, Section de Recherche, UMR IC/CNRS 168, 11 rue Pierre et Marie Curie, 75231 Paris Cedex 05, France \\ ${ }^{2}$ ESPCI, Laboratoire de Physico-Chimie Theorique, ESA CNRS 7083, 10 rue Vauquelin, 75231 Paris Cedex 05, France
}

\begin{abstract}
It is shown theoretically and experimentally that a liquid droplet can move on a surface structured with a locally asymmetric pattern when a breathing of the drop is induced by external means. Two different situations can be envisioned: a drop whose volume is modulated and a drop whose equilibrium contact angle is switched between two extreme values. This last case was experimentally investigated using electric fields acting on water droplets in castor oil. The main trends of the theory are verified although a quantitative analysis would necessitate either a simpler experimental geometry or a more elaborate model. The results are discussed with a miniaturization of the setup in mind which would have important potential applications in the field of integrated analysis systems.
\end{abstract}

PACS number(s): 47.65.+a, 05.45.-a, 06.60.Mr, 68.10.Cr

\section{INTRODUCTION}

The whole field of separation techniques has recently been rejuvenated by microtechnologies and the concepts of "laboratories on a chip." While addressing classical problems, miniaturized devices such as microchromatographs seem to hold the promise of an explosive development because of their intrinsic qualities: small amounts of material needed, very high separation velocities for the same efficiency compared with conventional devices, low costs of fabrication due to techniques widely used in microelectronics. In those microfluidic systems, typical channels have a width and depth of the order of a few tens of $\mu \mathrm{m}$ for a length which can go up to meters [1]. The quantities of product one has to deal with for an analysis become then very weak, typically of the order of a picoliter to a few nanoliters of solution. This last point can be very beneficial, for instance, in the field of DNA analysis where available quantities of product are very limited [2].

Paradoxically, although a major advantage of these techniques, the possibility of dealing with such small quantities of liquids has also become a difficult hurdle in their development. Being able to manipulate in a very controlled and precise way these very tiny droplets of aqueous solutions is in no way an easy task and some recent efforts have been precisely directed into that direction by different physical means: For instance, by Marangoni effect induced by thermal gradients [2], by a difference of pressure applied across a drop sitting on textured surfaces [2], or by an electrostatic traveling wave created by addressing microelectrodes underneath the drop [3].

In this paper, we present an original way of pumping liquid droplets which would be quite appropriate to help to solve these problems. The concepts we use here are derived from the family of "force-free", motion paradigms in which an object can be propelled without any macroscopic force or gradient in the direction of motion, by being submitted to a

\footnotetext{
*Present address: FAST, bat. 502, Universite d'Orsay, France
}

dissipative process in a locally asymmetric environment [4]. This symmetry based argument has been the subject of considerable interest in the past, partly due to its possible relevance as a frame work in which to understand the activity of motor proteins. Its value also stems from its ability to provide new ways to set colloidal particles [5] or more macroscopic [6] objects into motion in synthetic structures. Here, we demonstrate how a droplet can be set into motion when some internal shape modes are externally forced (by a vibration or a breathing of the drop) on a surface homogeneous and flat at a macroscopic scale but with a built-in polarity.

We first present a theoretical study of these ideas in two limiting cases and then an experimental macroscopic realization where the breathing of the drop is induced by an electrocapillary effect and which qualitatively validates our simple views on these phenomena. We end by extending these first results to situations dealing with microsystems.

\section{THEORETICAL PART: A LIQUID RIBBON ON A SAWTOOTHLIKE SURFACE}

\section{A. A liquid ribbon on a flat surface}

In partial wetting situations, a drop at equilibrium on a flat homogeneous solid surface takes the shape of a spherical cap. It is then entirely defined by its volume and its equilibrium contact angle $\theta_{e}$ resulting from a balance between the three involved interfacial tensions (Fig. 1). To get a simpler picture, we shall place ourselves in this section in a twodimensional (2D) geometry so that the drop is modeled by a ribbon of cross-section area $\Omega$. We neglect in the following gravity and sources of hysteresis other than the structure of the surface itself (for a review on the physics of wetting, see Ref. [7]).

\section{B. A steady ribbon on a sawtooth profile}

Consider now the same ribbon on a sawtooth periodic surface characterized by its period $\lambda$ and the angles $\alpha_{\text {max }}$ and $\alpha_{\min }\left(\alpha_{\max }>\alpha_{\min }\right)$ of the sides of the teeth with a horizontal 


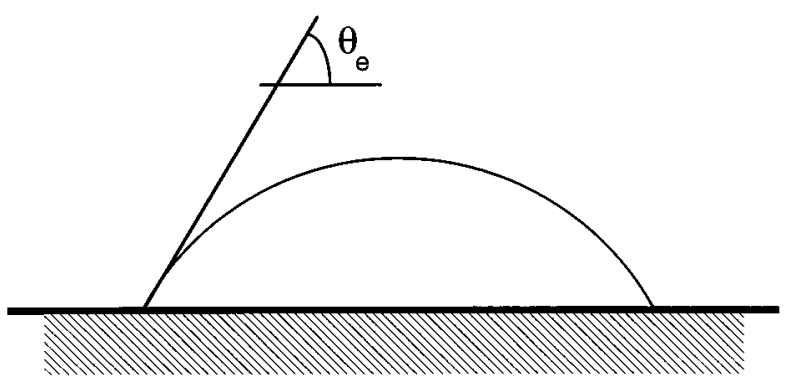

FIG. 1. Equilibrium shape of a liquid drop resting on a solid surface. The surrounding medium is either a gas or another liquid.

plane. Such a structure is represented in Fig. 2. We shall, in this paper, keep the conventions of this figure. We shall call $\theta$ the apparent contact angle (i.e., the angle of the drop with the horizontal plane). For a droplet much larger than $\lambda$, a stable (equilibrium) configuration generically corresponds to contact lines pinned on ridges [8]. The no-motion conditions then read

$$
\theta \geqslant \theta_{e}-\alpha_{\min }
$$

otherwise the left boundary would move to the right,

$$
\theta \leqslant \theta_{e}+\alpha_{\max }
$$

otherwise, the left boundary would move to the left,

$$
\theta \leqslant \theta_{e}+\alpha_{\min }
$$

otherwise the right boundary would move to the right,

$$
\theta \geqslant \theta_{e}-\alpha_{\max }
$$

otherwise the right boundary would move to the left.

The drop can thus be modeled as resting on a flat surface with a contact angle $\theta$ bound in angular sectors of the same aperture $\alpha_{\text {min }}+\alpha_{\max }$ but which have different tilts relative to the horizontal: $\theta_{e}-\alpha_{\max }$ on the right side and $\theta_{e}-\alpha_{\min }$ on the left side (see Fig. 3).

Note that there may be many equilibrium configurations characterized by $\theta$ verifying Eqs. (1)-(4) and the volume conservation which relates it to the number $n$ of teeth covered by the drop (neglecting the amount of liquid trapped in the teeth):

$$
\Omega \cong \frac{(n \lambda / 2)^{2}(\theta-\sin 2 \theta / 2)}{\sin ^{2} \theta}
$$

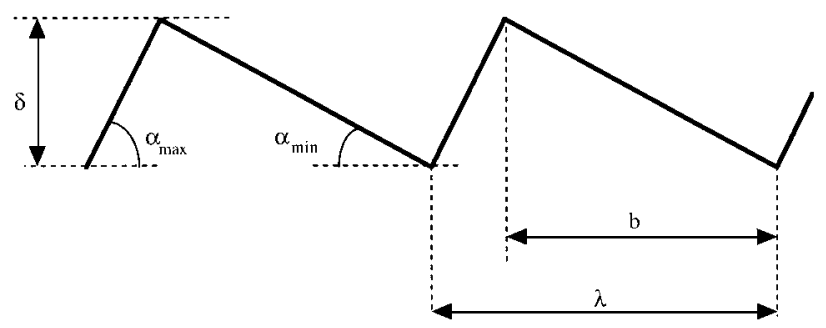

FIG. 2. Periodical sawtooth surface exhibiting a local but nonmacroscopic polarity.

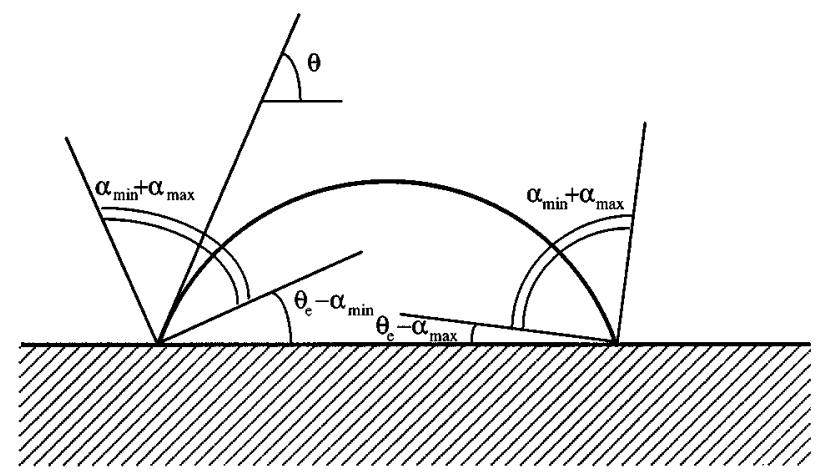

FIG. 3. When resting on a surface such as Fig. 2, the contact angle of the drop is confined into angular sectors. These sectors are different on both sides because of the polarity of the surface.

where $\lambda$ is the period of the pattern, related to the height $\delta$ of such a tooth by $\lambda / \delta=\left(\operatorname{tg} \alpha_{\max }\right)^{-1}+\left(\operatorname{tg} \alpha_{\min }\right)^{-1}$ (Fig. 2). If $\Omega$ (and $n$ ) is large, the increment $\delta \theta$ between two consecutive equilibrium angles will be quite small. We will restrict ourselves to this limit in the following.

\section{A breathing liquid ribbon on a sawtooth profile}

We will now show that a ribbon on a surface as described in the preceding paragraph can be set into directional motion if a breathing is induced by external means. To be more specific, we shall describe two very different physical situations: In the first case, the volume of the drop is cycling between two values at a constant equilibrium contact angle, whereas, in the second case, the drop is kept at constant volume and the equilibrium contact angle is modified by external action.

We also consider a slow breathing cycle so that $\theta$ has time during each interval to reach the closest equilibrium position, Eqs. (1)-(5), and is then pinned there until the next switch. In other words, we consider that the drop always has the time to reach an equilibrium shape (i.e., a cylindrical cap), the two contact angles on the left and on the right sides having the same value.

\section{Constant equilibrium contact angle}

One can consider this situation as a drop deposited on a sawtooth surface and having its liquid periodically pumped out and injected in (for instance, by evaporationcondensation processes or manually by carefully pumping and injecting liquid with a syringe). In that case, the positions of the two angular sectors are unchanged during this process (they only depend on the geometrical characteristics of the surface and on $\theta_{e}$ ) but the contact angle $\theta$ has to mirror the changes of volume in a way described by Eq. (5). We call $\Omega_{+}$and $\Omega_{-}$the maximum and minimum values of the volume in each cycle and $\tau$ the duration of one such cycle.

In the pumping step, $\theta$ decreases until it reaches the value of $\theta_{e}-\alpha_{\min }$ at which point it keeps this value and the left contact line moves to the right to another equilibrium position [Eq. (1)], the area covered by the drop evolving in a way described by Eq. (5). This process stops only when the pumping does. If one now increases the drop volume, $\theta$ increases accordingly up to the point where it reaches the value of $\theta_{e}+\alpha_{\min }$. It then keeps this value and the right contact 
line jumps to the right to its next equilibrium position [Eq. (3)]. Again, the area covered by the drop is given by Eq. (5). During this process, corresponding to one cycle of the drop's breathing, the two contact lines thus jump successively in the same direction. The drop as a whole thus moves accordingly. By cycling this process, an overall motion directed to the right with our conventions can thus be obtained.

The distance traveled per time cycle is $L=\left(n_{+}-n_{-}\right) \lambda$ where $n_{+}$(respectively $n_{-}$) is related to $\Omega_{+}$(respectively $\Omega_{-}$) by using $\theta=\theta_{e}+\alpha_{\min }$ (respectively $\theta_{e}-\alpha_{\min }$ ) in Eq. (5). The drop's velocity is then given by $V=L / \tau$.

\section{Constant volume}

If the equilibrium contact angle $\theta_{e}$ is periodically forced between two values $\theta_{e^{+}}$and $\theta_{e-}\left(\theta_{e^{-}}<\theta_{e^{+}}\right)$, the situation is then quite different. The possible means to practically achieve such a situation are less obvious and are described in the following.

We start with the situation depicted in Fig. 3 and we imagine an increase of the equilibrium contact angle $\theta_{e}$. This translates into a rotation of the authorized angular sectors clockwise for the right triple line, counterclockwise for the left one. Their apertures, being independent of $\theta_{e}$, stay unchanged. During this first step, $\theta$ keeps its current value. However, when $\theta_{e}-\alpha_{\min }$ hits the value of $\theta$, then the left contact line of the drop moves to the right until it reaches a stable position [given the volume conservation (5)] and $\theta$ then takes the value of $\theta_{e}-\alpha_{\min }$. This goes on as long as $\theta_{e}$ increases up to its final value $\theta_{e+}$. At that point, the value of the apparent contact angle $\theta$ is $\theta_{e+}-\alpha_{\min }$.

Conversely, when the equilibrium contact angle decreases, the two angular sectors now rotate in the opposite directions until $\theta_{e}+\alpha_{\min }$ reaches the value of $\theta_{e+}-\alpha_{\min }$ at which point the right contact line jumps to the right to its next equilibrium position. Again, this process continues until $\theta_{e}$ finally reaches the value of $\theta_{e_{-}}$. The apparent contact angle then takes the value of $\theta_{e_{-}}+\alpha_{\min }$.

Again, in this regime, the droplet center of mass thus clearly moves to the right as $\theta_{e}$ cycles. The distance traveled per time cycle is $L=\left(n_{-}-n_{+}\right) \lambda$ where $n_{-}$(respectively $n_{+}$) is related to $\theta_{e-}$ (respectively $\theta_{e+}$ ) by using $\theta=\theta_{e+}$ $-\alpha_{\text {min }}$ (respectively $\theta_{e^{-}}+\alpha_{\text {min }}$ ) in Eq. (5). If we call $\tau$ the duration of a complete cycle, the velocity is $L / \tau$. However, in a more general situation, both boundaries may move at the same time, although a net average drift would remain.

Note that we have implicitly assumed that

$$
\theta_{e+}-\alpha_{\min }>\theta_{e^{-}}+\alpha_{\min },
$$

which is a necessary condition to set the drop into motion.

In the same line, if the contact angle is changed suddenly between the two extreme values $\theta_{e+}$ and $\theta_{e_{-}}$(instead of progressively), another condition is required for only one of the contact lines to move at a given moment, namely, $\theta_{e_{-}}$ $+\alpha_{\max }>\theta_{e+}-\alpha_{\min }$.

More generally, as long as Eq. (6) holds so that motion is induced, one expects that the drop drifts on average.

\section{A few words of caution}

At this point, let us describe some imperfections of this simple model.
First, we have dealt with a liquid ribbon, however, these objects are prone to the Rayleigh instability [9] and thus cannot be produced in the real world. However, this instability should be largely saturated by the pinning of the triple lines on the discontinuities of the surface.

Second, and it is a consequence of the first caveat of the model, since we are dealing with 3D drops, the motion of the drop in the transverse direction (parallel to the ridges) is going to be much easier than having to cross the ridges and valleys represented by the structured surface. This effect seriously limits the amplitude of the cyclic motion of the contact line along the sawtooth profile and thus the efficiency of the above mechanism. However, confining the drop in a channel perpendicular to the structure should overcome this difficulty.

Of course, no motion can ever happen if the switching from 1 to 2 is unable to unpin the contact line, i.e., if there exist angle $\theta$ solutions of Eqs. (1)-(4) for both $\Omega_{+}$and $\Omega_{-}$ in case I and $\theta_{e+}$ and $\theta_{e_{-}}$in case II. Similarly, the value of $\tau$ is of importance, and many regimes in which it is short enough so that the contact lines do not reach equilibrium could be investigated (the typical time scale is $L \eta / \gamma$ where $\eta$ and $\gamma$ are the viscosity and the surface tension of the liquid).

One has also to make sure that the liquid actually fills in the valleys. As a matter of fact, the surrounding medium has been shown to tend to stay trapped in the troughs in very similar geometries $[7(\mathrm{~b}), 10]$.

Finally, we have neglected hysteresis of the contact angle (on the unstructured surface) in the analysis of the wettingdewetting process. Taking it into account adds further constraints to the geometric ones described above.

\section{ELECTROCAPILLARITY: A WAY OF INDUCING PERIODIC PERTURBATIONS ON DROPS}

In this section, we investigate the effects of an electric field on drops as a way to induce the breathing necessary for the motion.

\section{A. Isolated drops}

Let us first consider a drop of liquid of dielectric constant $\epsilon_{1}$ and resistivity $\sigma_{1}$ placed between the two plates of a capacitor. The medium inside the capacitor is itself a liquid whose dielectric constant and resistivity are hereafter denoted $\epsilon_{m}$ and $\sigma_{m}$. For the sake of clarity, we shall now focus on a water droplet in a capacitor whose medium is an oil. To avoid a possible electrical contact of the drop with the plates of the capacitor, they are coated with an insulating film whose electrical characteristics are assumed to be identical to those of the liquid medium [Fig. 4(a)]. The viscosities are $\eta_{1}$ and $\eta_{m}$, and the water/oil interfacial tension is $\gamma$. The different physical constants used in this paper are recapitulated in Table I.

Upon the application of an oscillatory electric field, the drop will deform. We call this deformation: $D=\left(d_{1}\right.$ $\left.-d_{2}\right) /\left(d_{1}+d_{2}\right)\left(d_{1}\right.$ and $d_{2}$ being the half values of the long and short axis) [Fig. 4(b)]. We will restrict ourselves to the small deformation regime: $D \cong\left(d_{1}-d_{2}\right) / 2 a$ where $a$ is the drop radius.

We first qualitatively treat this problem in a quasidimensional way by writing a dynamical equation for the equilib- 


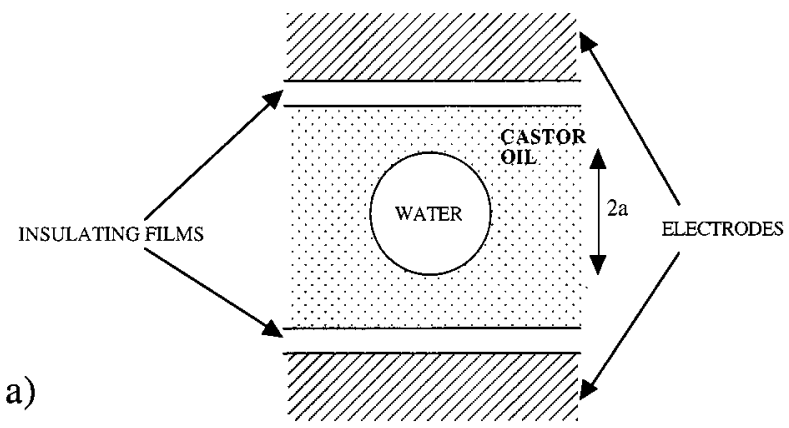

b)

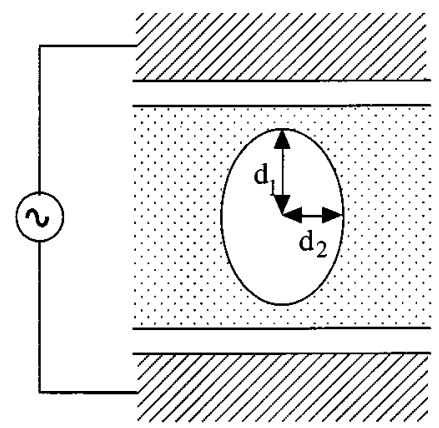

FIG. 4. A suspended drop of water in castor oil elongates in the direction of the applied field when submitted to an ac voltage.

rium of forces (we consider here that there is only one characteristic length in the problem, namely, the radius of the drop $a$ ). Let us note that, in such an analysis, we can deal only with effective physical constants such as $\eta$ and $\epsilon$ which we cannot, at this level, relate to their water or oil counterparts. The contributions which have to be taken into account are

(1) the electrostatic pressure is proportional to $\epsilon \mathbf{E}^{2}$ thus yielding a force proportional to $a^{2} \varepsilon \mathbf{E}^{2}$

(2) the Laplace pressure tends to restore the drop to its spherical shape; its expression is $\gamma\left(1 / d_{1}-1 / d_{2}\right)$, and acting on an object of size $a$, this leads to a force $\gamma a^{2}\left(1 / d_{1}\right.$ $\left.-1 / d_{2}\right)$

(3) the rate of the velocity of deformation being $\partial_{t} D$, the dissipation by volume unit is thus $\eta\left(\partial_{t} D\right)^{2}$. In the volume of the drop, we thus get a dissipation $T \dot{S}=a^{3} \eta\left(\partial_{t} D\right)^{2}$. To get the corresponding force, we interpret this expression in the form of $T \dot{S}=F V$ where $V=a\left(\partial_{t} D\right)$ and we thus get $F$ $=a^{2} \eta \partial_{t} D$.

Balancing these three contributions, we get

$$
\eta a \partial_{t}(D a)=\gamma K_{1}\left(\frac{1}{d_{1}}-\frac{1}{d_{2}}\right) a^{2}+K_{2} \varepsilon E^{2} a^{2},
$$

$K_{1}$ and $K_{2}$ being numerical constants.
After some calculations, one eventually gets, in the case of an oscillating electric field $E=E_{0} \cos (\omega t)$,

$$
\begin{gathered}
D \cong K \frac{\varepsilon a E_{0}^{2}}{\gamma}\left[1+\frac{1}{\sqrt{1+\omega^{2} \tau^{2}}} \cos (2 \omega t+\varphi)\right] \\
\text { with } \tau \approx \frac{\eta a}{\gamma} \text { and } \cos \varphi=\frac{1}{\sqrt{1+\omega^{2} \tau^{2}}} .
\end{gathered}
$$

As a matter of fact, the deformations of such isolated drops have already been thoroughly studied experimentally and theoretically in particular in Refs. $[11,12]$. In particular, when subjected to electric fields of the order of a few $\mathrm{kV}$ $\mathrm{cm}^{-1}$, water drops in castor oil are elongated in the direction of the field in a way schematically represented in Fig. 4(b). On the theoretical side, a very general discussion yielding to complex formulas to characterize this deformation can be found in [11]. However, in our case, given the typical values of conductivities, dielectric constants, and viscosities dealt with in this paper (see Table I), these expressions can be considerably simplified and one finds

$$
D=\frac{d_{1}-d_{2}}{d_{1}+d_{2}}=D_{s}+D_{0}
$$

where

$$
D_{s}=\frac{9 \varepsilon_{m}}{16 \gamma} a E^{2}
$$

is the steady deformation, and

$$
\begin{aligned}
& D_{0}=D_{s}\left[\frac{\cos (2 \omega t+\beta)}{\left(1+4 \omega^{2} a^{2} \eta_{m}^{4} / \gamma^{2} \eta_{l}^{2}\right)^{1 / 2}}\right], \\
& \text { with } \cos \beta=\left(1+4 \frac{\omega^{2} a^{2} \eta_{m}^{4}}{\gamma^{2} \eta_{l}^{2}}\right)^{-1 / 2}
\end{aligned}
$$

is the time-dependent deformation.

Equation (11) is indeed remarkably similar to Eq. (8) and thus confirms the rough but simple analysis leading to this equation. We will thus empirically identify $\varepsilon$ to $\varepsilon_{m}$ and $\eta$ to $2 \eta_{m}^{2} / \eta_{1}$.

Although for frequencies larger than $\omega_{0}=\gamma \eta_{1} / 2 a \eta_{m}^{2}$, the oscillatory response of the drop will be attenuated, we have here a simple way of soliciting it with a low frequency ac field.

\section{B. Drops sitting on surfaces}

If the drop is now placed on the film covering one of the plates of the capacitor (Fig. 5), the interface between the

TABLE I. Physical constants of the liquids used in the present study.

$\left.\begin{array}{ccccc}\hline \hline & & \text { Relative } & \\ & \text { Conductivity } & \text { permittivity } & \text { Viscosity } & \text { Interfacial tension } \\ \hline \text { Water } & 0.5 \mu \mathrm{S} \mathrm{cm}^{-1} & 80 & 10^{-3} \mathrm{~Pa} \mathrm{~s} \\ \text { Castor oil } & <0.1 \mathrm{pS} \mathrm{cm}{ }^{-1} & 4.5 & 1.4 \mathrm{~Pa} \mathrm{~s}\end{array}\right\}$




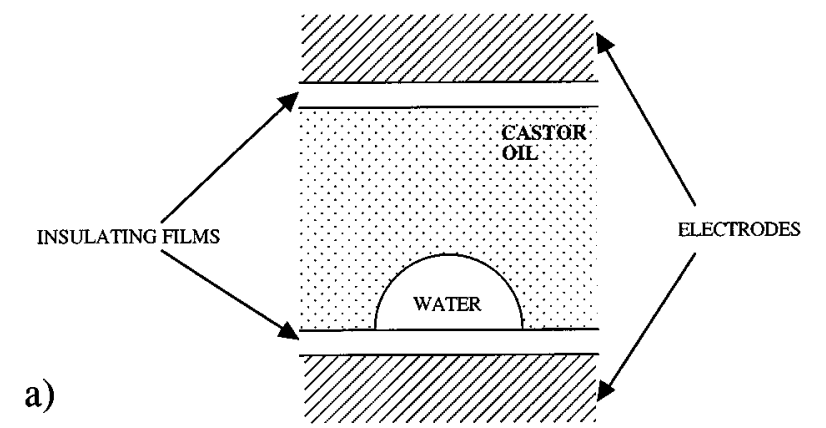

b)

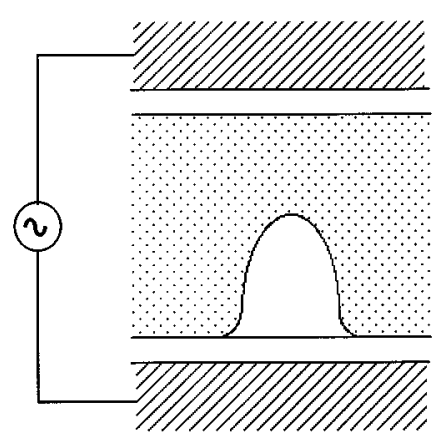

FIG. 5. If the drop of Fig. 4 is resting on a solid surface, the application of the electric field makes it simultaneously spread on the surface and elongate toward the other electrode.

water drop and the plate of the capacitor must also be taken into account. To minimize the electrostatic energy of the capacitor, it is advantageous for the drop to maximize its surface of contact with the plate and this situation thus leads to a spreading of the drop on the solid surface [13]. To see that more clearly, just consider the capacitor made of the following sandwich: (one of the metallic plates)/(the insulating film)/[the water drop (considered here as the second plate of this capacitor)]. To minimize the electrostatic energy, it is favorable to increase the value of this capacitance; this corresponds to a decrease of the effective surface tension of the drop and thus to its spreading. This effect, often called electrowetting, is described in more detail in Ref. [13].

We can integrate this effect in our model by adding to the different terms of Eq. (10) the contribution of the surface [again, our treatment implies that $a$ is the only characteristic length of the drop, meaning that its height and its radius are both comparable (not too small contact angles)]

(1) The electrostatic term takes into account the effect described above and now includes a term of the form $a d \varepsilon E^{2}$ where $D$ is the insulating film thickness (the permittivity of this film is supposed identical to that of the medium).

(2) The Laplace pressure term should now include a new term of the form $\gamma a\left(\cos \theta_{e}-\cos \theta\right) \cong a \gamma D$ which is then very similar to the Laplace term in the free drop case.

(3) As for the dissipation near the line, its expression is classically given by $a \eta\left(\partial_{t}(a D)\right)^{2} / \theta$ [7], yielding a surface induced friction force: $a \eta\left(\partial_{t}(a D)\right) / \theta$, again similar to its expression in the absence of surface.

We therefore conclude that the surface contribution mainly appears in the electrostatic pressure term. By analogy with the preceding calculation, we get the following expression of the deformation with regard to the equilibrium position:

$$
\begin{gathered}
D=\left(K_{1}^{\prime}+K_{2}^{\prime} \frac{d}{a}\right) \frac{\varepsilon a E_{0}^{2}}{\gamma}\left(1+\frac{1}{\sqrt{1+\omega^{2} \tau^{2}}} \cos (2 \omega t+\varphi)\right), \\
\text { with } \tau \approx \frac{\eta a}{\gamma} \text { and } \cos \varphi=\frac{1}{\sqrt{1+\omega^{2} \tau^{2}}} .
\end{gathered}
$$

By using a low frequency voltage between the plates of the capacitor, one can thus induce the breathing of a drop deposited on one of the plates. This breathing occurs at a frequency twice as large as that of the imposed electric field. This is also true if the drop bridges the two plates.

Let us note at this point that we have computed here the behavior of spherical drops under field and not that of the infinite ribbons dealt with in Sec. I. However, we expect the qualitative behaviors to be similar in these two situations although the numerical constants should be different. As a matter of fact, the scaling analysis is similar for both geometries.

We thus have here one of the two ingredients necessary to motion according to the analysis of the first section of this paper. The structuring of the surface is the other ingredient and is described in the next section.

\section{MOTION OF DROPS ON STRUCTURED SURFACES}

We have just seen in the preceding section that oscillations of a drop can be induced by the application of an ac electric field. According to the model presented in our first section, such breathing drops are expected to be set into motion on a surface presenting a periodic structure whose elementary pattern is asymmetric. In our terminology, it is the case of a constant volume drop at a variable contact angle theoretically described in Sec. IC 2.

\section{A. Structuring of the surfaces}

One or both of the insulating films covering the plates are structured in the following way.

We first fabricate a "master" by annealing above their glass transition a pack of microscope glass cover slips (160 $\mu \mathrm{m}$ thick) stacked against an inclined plane such as in Fig. 6(a). Practically, we used an annealing time of $2 \mathrm{~h}$ at $600^{\circ} \mathrm{C}$. We then softly imprint the shape of such a stack on a paraffinlike film (Parafilm, American Can, thickness $\cong 200 \mu \mathrm{m}$ ) previously placed on the electrode [Fig. 6(b)]. For a good imprinting, the film needs to be somewhat softened by a slight heating using a heating plate around $70^{\circ} \mathrm{C}$.

By modifying the angle at which the master is annealed, one can have control over the period and the asymmetry of the structure imprinted on the surface. In the following, surfaces will be denoted by their period $(\lambda)$ and asymmetry ( $x$ $=b / \lambda$ ) (see Fig. 2). However, since the thickness of the coverslips used to fabricate the master is fixed $(160 \mu \mathrm{m}$ in our case), $\lambda$ and $x$ are not independent. Other sets of parameters can be easily fabricated, however, by using cover slips of different thickness. 


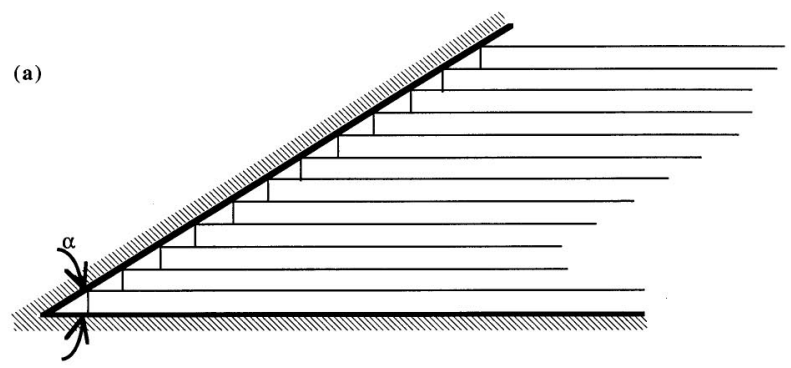

(b)
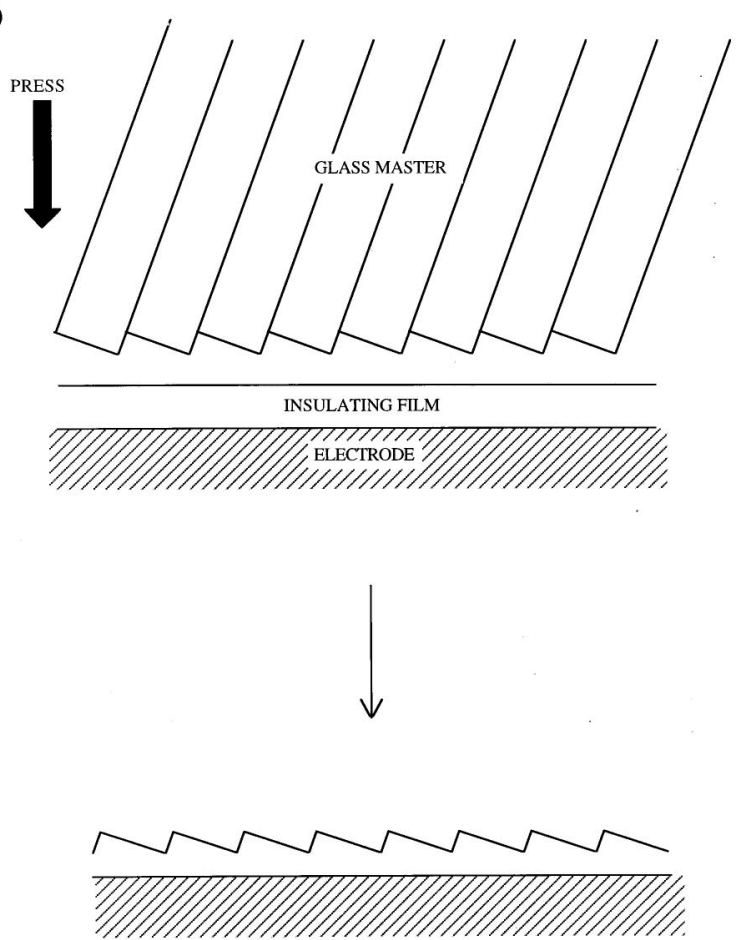

(c)

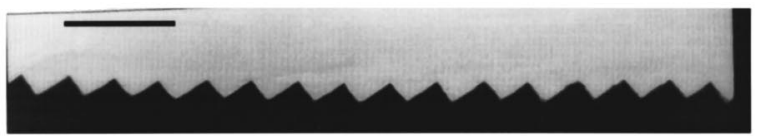

FIG. 6. (a) Fabrication of the "master" by stacking of glass cover slips. (b) Imprinting of the master to get a sawtooth surface. (c) Final surface on the insulating film. Bar $=0.5 \mathrm{~mm}$.

\section{B. Experimental setup}

The two plates of the capacitor are then placed in a specially designed cell which enables us to dynamically follow the profile of the drop under the influence of the electrical field. Once the cuvette is filled with castor oil (Prolabo, France), a drop of deionized water is placed on the bottom surface. The cell is then closed and, depending on the volume of the drop, it can either touch only one of the two plates or bridge them together. We consider in this paper only drops bridging the two surfaces. The horizontality of the cell was checked with a bubble level. Typical drops' sizes are of a few millimeters (i.e., typical volumes of $10 \mu \mathrm{l}$ ).

An electric field was then applied between the two electrodes by amplifying the ac voltage of the mains $(220 \mathrm{~V}, 50$ $\mathrm{Hz}$ ) up to $1.6 \mathrm{kV}$. The distance between the plates can be varied between 1 and $10 \mathrm{~mm}$.
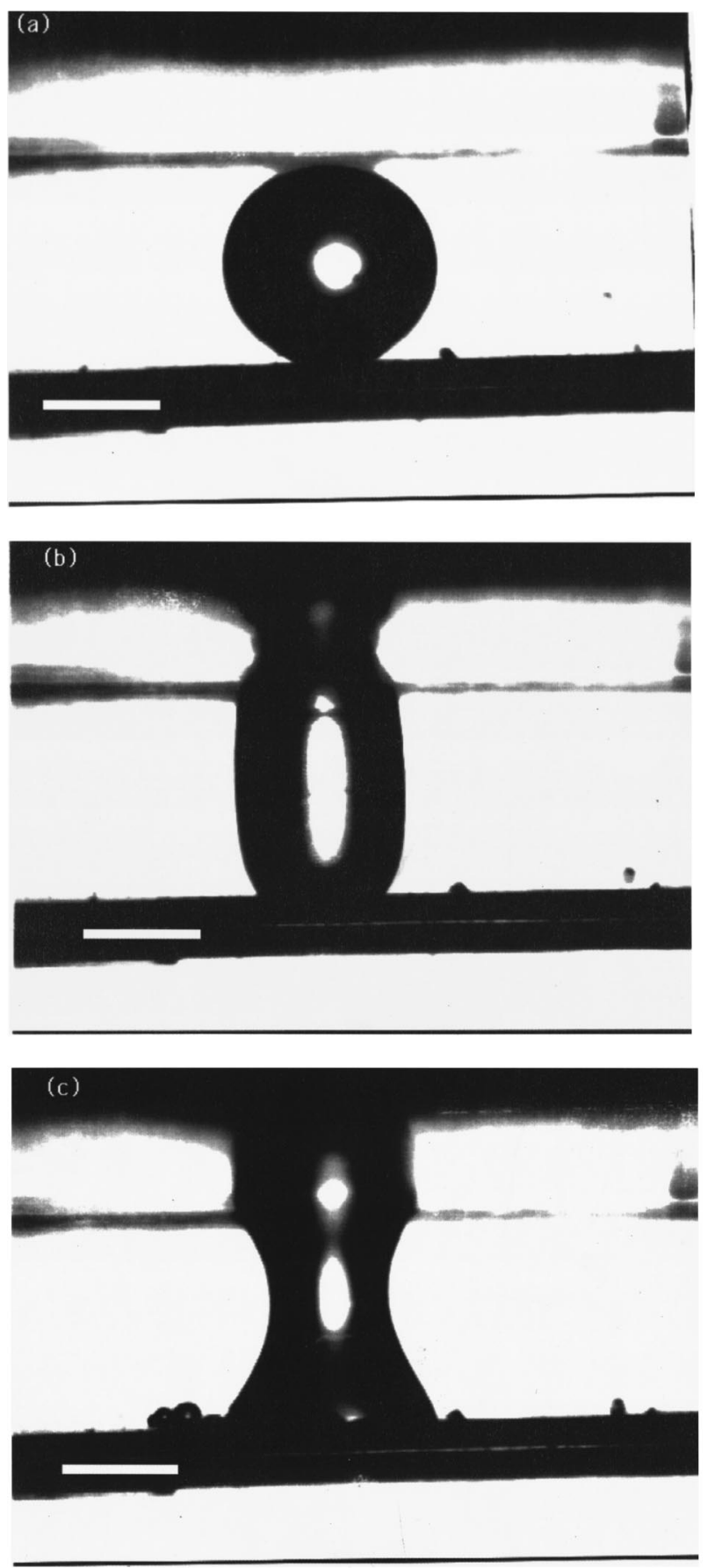

FIG. 7. Side view of a droplet between two electrodes bearing $240 \mu \mathrm{m}$ flat insulating films upon the application of a $50 \mathrm{~Hz}$ electric voltage $E$ : (a) $E=0$; (b) $E=840 \mathrm{~V}$; (c) $E=1200 \mathrm{~V}$. The bar represents $1 \mathrm{~mm}$.

Side views of the drop could be observed through a flat window fixed up on the cell by using a low magnification binocular equipped with a charge coupled device (CCD) camera. They are then recorded on a VCR for analysis.

\section{Motion of drops: results and discussion}

When bridging two flat films, the drop deforms rapidly when submitted to the electric field. As expected, it becomes elongated in the direction of the field while its contact angle decreases drastically. As can be observed in Fig. 7, where 

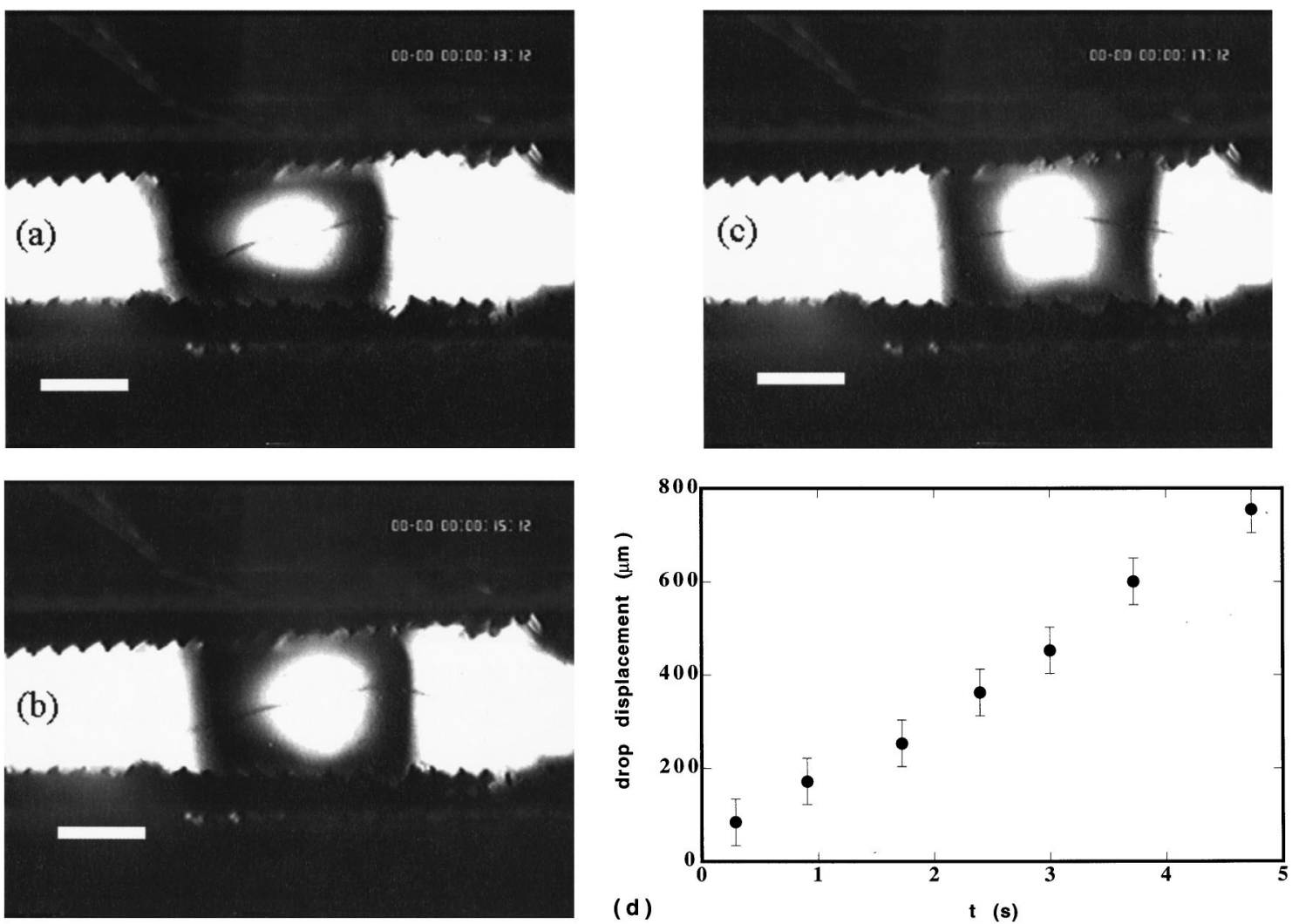

FIG. 8. (a)-(c) Motion of a drop squeezed between two structured surfaces upon the application of a $700 \mathrm{~V} 50 \mathrm{~Hz}$ electric voltage. The time interval between two snapshots is $2 \mathrm{~s}$. The bar represents $1 \mathrm{~mm}$ (grating $\lambda=190 \mu \mathrm{m}, x=0.7$ ). Defects on the window of the cell as well as the relatively narrow depth of field of our focusing setup induce some optical artifacts such as an apparently blurry grating or the bump on the right hand side of the photographs. (d) Position of the drop as a function of time. This uniform motion is the stationary regime often preceded by an acceleration regime during several seconds (not shown).

fields up to $6 \mathrm{kV} \mathrm{cm}^{-1}(50 \mathrm{~Hz})$ were applied, these deformations and the associated contact angle variations can be very large.

When the drop bridges two structured films similarly oriented, a deformation of similar amplitude occurs. However, on top of this deformation, an overall motion of the drop is observed. For instance, on surfaces $\lambda=190 \mu \mathrm{m}, x=0.7$, the drop moves at $0.15 \mathrm{~mm} \mathrm{~s}^{-1}$ for field values of $4700 \mathrm{~V} \mathrm{~cm}^{-1}$ acting on a drop squeezed between two plates separated by $1.5 \mathrm{~mm}$ (see Fig. 8). As for the direction of this motion, it is in accordance with the theoretical predictions (Sec. IC 2).

Apart from the effect described in the theoretical part, we are aware that several artifacts can cause such a motion. An imperfect horizontality can be ruled out as results stay consistent when the orientation of the whole cell is reversed. We have also checked for the presence of a macroscopic angle between the two electrodes and could not measure any by optical means (meaning that it is smaller than a few mrad). We have also performed the following control experiments.

(1) The observations are invariant by any permutation of the plates in the cell provided their polarities are kept identically oriented.

(2) When using two gratings oriented in opposite directions, no motion is observed. The $S$ shape taken by the drop (see Fig. 9) is then very significant of the ongoing pro- cess as its top and bottom parts tend to drive it in opposite directions.

(3) The drop does not move either when the experiment is repeated on a surface characterized by a symmetrical pattern $\left(x=0.5, \alpha=45^{\circ}\right)$.

(4) Finally, when only one of the two plates is structured, a motion is still observed but it is much less efficient (drops need higher voltages and go much slower).

If we come back to Eq. (12), we can easily be convinced that working at $50 \mathrm{~Hz}$ (a frequency we have chosen for ob-

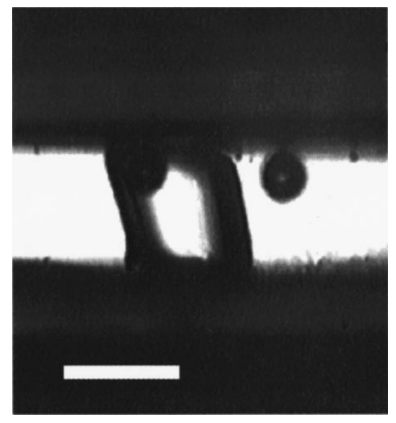

FIG. 9. Side view of a drop squeezed between two gratings oriented in opposite directions upon the application of an electric voltage $(50 \mathrm{~Hz}, 1600 \mathrm{~V}) . \mathrm{Bar}=1 \mathrm{~mm}, \lambda=190 \mu \mathrm{m}, x=0.7$. 
vious practical reasons) is a very poor choice in terms of efficiency of the motion. As a matter of fact, the cutoff frequency which appears in the description of the electrocapillary phenomena is $\omega_{0}=\gamma \eta_{1} / 2 a \eta_{m}^{2}$, which numerically comes close to $10^{-3} \mathrm{~Hz}$. By plugging the physical constants of our system (Table I) in Eq. (11), we get $D_{s} \cong 1$ and $D_{0}$ $\cong 10^{-3} D_{s}$. We thus mainly deal with a small periodic perturbation on top of a large static deformation. We see nevertheless that this very limited dynamic deformation is enough to put the drop into motion. Note that we have used here calculations performed for a spherical drop whereas the geometry dealt with in this paper is more complex, the drop being confined between the two electrodes. However, the dimensions of the setup (and, in particular, the interelectrode spacing) are comparable to the drop size. We thus expect our crude estimation to be reliable.

With our equipment, no direct observation of the dynamic deformation can be observed since we expect these movements of the drop to occur at $100 \mathrm{~Hz}$ whereas we are limited in our observations by the video frequency at $25 \mathrm{~Hz}$. In our films, the drop moves as a whole but we do not observe any breathing.

Given the velocity of $0.15 \mathrm{~mm} \mathrm{~s}^{-1}$ and a period of 190 $\mu \mathrm{m}$ for the structuring of the surface, we arrive at the conclusion that the drop moves at an average of one period every 200 time cycles. This is consistent with the very strong attenuation of the electric field we get at these frequencies. Drops in contact with only one structured surface then move slower just because the probability of step decreases accordingly.

We have also compared the motion of drops of different sizes between two structured plates and noted a general trend for the larger drops to move slower. Gravity is not a relevant parameter for these almost isodensity liquids; we thus attribute this behavior to a larger effective friction of the drop on the surface [since the principle of propulsion is a change in contact angles, it acts only on the triple line and should not depend on the drop's size (at least for ribbons)].

Although the model gives some track for a qualitative interpretation of our observations, we cannot go much further at this level primarily because, as was pointed out in Sec. IC3, the main displacement of the contact line is along the grooves of the grating and not perpendicular to it. In other words, the displacement is mainly perpendicular to the motion and our two-dimensional analysis obviously does not hold quantitatively.

Another strong hypothesis of the theoretical analysis lies in the fact that the drops are always supposed to be at equilibrium. Here, clearly, the drop moving by a fraction of period per time cycle, it is not the case.

These reasons thus make any quantitative comparison of theoretical and experimental velocities, for instance, out of the scope of our simple model. Nevertheless, it still describes correctly the main trends of the motion and, as such, it provides a useful framework for the analysis of the experiments and the design of future ones particularly at smaller scales.

\section{CONCLUSION}

We have shown both theoretically and experimentally that the motion of liquid drops can be induced by a structuring of the surface coupled with an ac electric field perpendicular to the direction of motion. This motion is another example of how local spatial asymmetry coupled with dissipation can lead to a macroscopic displacement.

This kind of induced motion on liquids can also have many practical applications as emphasized in the Introduction. The miniaturization of a device similar to the one described here should enable one to manipulate droplets of a few $100 \mathrm{pl}$ with teeth sizes of the order of $10 \mu \mathrm{m}$ easily achievable using ruling techniques used classically for the fabrication of diffraction gratings. By using more sophisticated techniques, we think that drops as small as a few $\mathrm{pl}$ can be handled.

As for the surface, one can play with the chemical composition rather than with the profile. Macroscopic gradients of temperature or chemical composition are known to set drops into motion [14]. We can think here to transpose these large scale gradients by local asymmetric modulations. We then come close conceptually to experiments in which the solute contained in organic droplets is able to react on the surface, therefore creating an asymmetry between the front and rear sides of the drop [15]. However, our setup would be more practical as the drop would not have to carry any reagent since we propose a permanent treatment of the surface.

However, these potential developments will need better control of the experiments in order to come closer to the ideal conditions described by the model. We just cite here a few points which can be easily improved.

(1) The motion of the contact line parallel to the grooves can be suppressed by confining the drop in a channel.

(2) The process itself of the fabrication of the grating can be easily improved by using a better master than the stacking of the glass slides.

(3) Using a faster camera should enable us to better characterize the $50 \mathrm{~Hz}$ breathing of the drop. Given the frequencies dealt with in this work, an image acquisition system working at about 200 (images) $\mathrm{s}^{-1}$ should be enough (providing it keeps a good resolution in order to observe the small ac deformations induced by the field).

(4) Finally, the frequency chosen for the experiments can be tuned and lower frequencies should enable us to get larger deformations and thus a greater efficiency of the process.

These points as well as some miniaturization developments are presently under study in our laboratory. In particular, experiments performed at low frequencies enable better observations of the pinning-unpinning processes and a closer correlation with the motion of the drops.

\section{ACKNOWLEDGMENTS}

We acknowledge financial support from a contrat coopératif Institut Curie/Montagne ste-Geneviève and from the thematic CNRS program Ultimatech. It is a pleasure to thank Dr. B. Berge, Dr. H. Biebuyck, Dr. R. Anderson, and Dr. A. De Mello for fruitful discussions. 
[1] C. S. Effenhauser, A. Manz, and H. M. Widmer, Anal. Chem. 67, 2284 (1995); E. Kim, Y. Xia, G. M. Whitesides, J. Am. Chem. Soc. 118, 5722 (1996); E. Delamarche, A. Bernard, H. Schmid, B. Michel, and H. Biebuyck, Science 276, 779 (1997). See also Micro Total Analysis Systems '98, edited by D. J. Harrison and A. Van Den Berg (Kluwer, Dordrecht, 1998).

[2] M. A. Burns, C. H. Mastrangelo, T. S. Sammarco, F. P. Man, J. R. Webster, B. N. Johnson, B. Foerster, D. Jones, Y. Fields, A. R. Kaiser, and D. T. Burke, Proc. Natl. Acad. Sci. USA 93, 5556 (1996); M. A. Burns, B. N. Johnson, S. N. Brahmasandra, K. Handique, J. R. Webster, M. Krishnan, T. S. Sammarco, P. M. Man, D. Jones, D. Helsinger, C. H. Mastrangelo, and D. T. Burke, Science 282, 484 (1998); R. L. Columbus, U.S. Patent No. 4233029 (1986).

[3] M. Washizu, IEEE Trans. Ind. Appl. 34, 732 (1998).

[4] P. Hänggi and R. Bartussek, in Nonlinear Physics of Complex Systems, edited by J. Parisi, S. C. Müller, and W. Zimmermann (Springer, Berlin, 1996), p. 294; F. Jülicher, A. Ajdari, and J. Prost, Rev. Mod. Phys. 69, 1269 (1997); D. Astumian, Science 276, 917 (1997).

[5] A. Ajdari and J. Prost, C. R. Acad. Sci., Ser. II: Mec. Phys., Chim., Sci. Terre Univers 315, 1635 (1992); J. Rousselet, L. Salomé, A. Ajdari, and J. Prost, Nature (London) 370, 446 (1994); L. P. Faucheux, L. S. Bourdieu, P. D. Kaplan, and A. J. Libchaber, Phys. Rev. Lett. 74, 1504 (1995); L. Gorre-
Talini, S. Jeanjean, and P. Silberzan, Phys. Rev. E 56, 2025 (1997).

[6] A. Ajdari, J. Phys. I 4, 1577 (1995); L. Gorre-Talini, E. Ioannidis, and P. Silberzan, Europhys. Lett. 33, 267 (1996).

[7] (a) A. Adamson, Physical Chemistry of Surfaces (Wiley, New York, 1982); (b) P. G. De Gennes, Rev. Mod. Phys. 57, 827 (1985).

[8] J. F. Oliver, C. Huh, and S. G. Mason, J. Colloid Interface Sci. 59, 568 (1977).

[9] K. Sekimoto, R. Oguna, and K. Kawasaki, Ann. Phys. (N.Y.) 176, 359 (1987).

[10] R. Dettre and R. Johnson, in Contact Angle, Wettability and Adhesion, edited by F. M. Fowkes, Advances in Chemistry Series No. 43 (American Chemical Society, Washington, DC, 1964).

[11] O. Vizika and D. A. Saville, J. Fluid Mech. 239, 1 (1992).

[12] G. Taylor, Proc. R. Soc. London, Ser. A 291, 159 (1966).

[13] B. Berge, C. R. Acad. Sci., Ser. II: Mec., Phys., Chim., Sci. Terre Univers 317, 157 (1993).

[14] T. Ondarçuhu and M. Veyssié, J. Phys. II 1, 75 (1991); M. Chaudhury, and G. M. Whitesides, Science 256, 1539 (1992); J. B. Brzoska, F. Brochard-Wyart, and F. Rondelez, Langmuir 9, 2220 (1993).

[15] F. D. Dos Santos and T. Ondarçuhu, Phys. Rev. Lett. 75, 2972 (1995). 